\section{GW23-e1481 PAINLESS AORTIC DISSECTION WITH INITIAL SYMPTOMS OF PARAPLEGIA AND ACUTE RENAL FAILURE: A CASE REPORT}

doi:10.1136/heartjnl-2012-302920u.4

Zheng Ziyu, Ye Zi, Ye Jialin, Wang Weiping, Zhan Hong. Department of Emergency, The First Affiliated Hospital of Sun Yat-sen University

Objectives A 67-year-old man was transferred to the Emergency Department of our hospital for emergent evaluation of paraplegia and oliguria, from the local hospital of the nearby town, where he was admitted complaining from sudden, painless, progressive bilateral leg weakness and oliguria 4 days earlier. He gave no history of hypertension, diabetes mellitus or hyperlipidaemia, and had a negative family history of aortic diseases. On initial evaluation, the patient had a blood pressure of $131 / 71 \mathrm{~mm} \mathrm{Hg}$. His oral temperature was $36.4^{\circ} \mathrm{C}$, pulse rate was 82 beats $/ \mathrm{min}$ and respiratory rate was $20 / \mathrm{min}$. He presented complete flaccid paraplegia with oliguria (urinary output $<400 \mathrm{ml} / \mathrm{d}$ ) and urinary retention, loss of pain and temperature sensation, vibration and position sense below the TH7 level bilaterally. Other general physical examinations were unremarkable. Laboratory tests showed a white blood cell count of $19.80 \times 10^{9} / 1$, haemoglobin concentration of $109 \mathrm{~g} / \mathrm{l}$, blood urea nitrogen concentration of $50 \mathrm{mmol} / \mathrm{l}$, blood creatinine concentration of $820 \mu \mathrm{mol} / \mathrm{l}$, sodium concentration of $114 \mathrm{mmol} / \mathrm{l}$, and potassium concentration of $4.6 \mathrm{mmol} / \mathrm{l}$. The liver function tests were normal and other observations were unremarkable. Later thoracic and lumbar MRI revealed swelling of thoracolumbar spinal cord, with no enhancement on T1-weighted images (wi) and increased signal on T2-wi at the TH9-TH12 levels, suggesting cord ischaemia. At the same MR sequences, the double lumen of the descending aorta involving bilateral renal arteries indicated dissection in both sagittal and axial images. The diagnosis of Stanford type B acute aortic dissection was confirmed. When patients present with or develop signs and symptoms of paraplegia without obvious cause, aortic dissection should be considered, even without the presence of characteristic thoracic pain.

Methods A 67-year-old man was transferred to the Emergency Department of our hospital for emergent evaluation of paraplegia and oliguria, from the local hospital of the nearby town, where he was admitted complaining from sudden, painless, progressive bilateral leg weakness and oliguria 4 days earlier. He gave no history of hypertension, diabetes mellitus or hyperlipidaemia, and had a negative family history of aortic diseases. On initial evaluation, the patient had a blood pressure of $131 / 71 \mathrm{~mm} \mathrm{Hg}$. His oral temperature was $36.4^{\circ} \mathrm{C}$, pulse rate was 82 beats $/ \mathrm{min}$ and respiratory rate was $20 / \mathrm{min}$. He presented complete flaccid paraplegia with oliguria (urinary output $<400 \mathrm{ml} / \mathrm{d}$ ) and urinary retention, loss of pain and temperature sensation, vibration and position sense below the TH7 level bilaterally. Other general physical examinations were unremarkable. Laboratory tests showed a white blood cell count of $19.80 \times 10^{9} / 1$, haemoglobin concentration of $109 \mathrm{~g} / 1$, blood urea nitrogen concentration of $50 \mathrm{mmol} / \mathrm{l}$, blood creatinine concentration of $820 \mu \mathrm{mol} / \mathrm{l}$, sodium concentration of $114 \mathrm{mmol} / \mathrm{l}$, and potassium concentration of $4.6 \mathrm{mmol} / \mathrm{l}$. The liver function tests were normal and other observations were unremarkable. Later thoracic and lumbar MRI revealed swelling of thoracolumbar spinal cord, with no enhancement on T1-weighted images (wi) and increased signal on T2-wi at the TH9-TH12 levels, suggesting cord ischaemia. At the same MR sequences, the double lumen of the descending aorta involving bilateral renal arteries indicated dissection in both sagittal and axial images. The diagnosis of Stanford type B acute aortic dissection was confirmed. When patients present with or develop signs and symptoms of paraplegia without obvious cause, aortic dissection should be considered, even without the presence of characteristic thoracic pain.

Results A 67-year-old man was transferred to the Emergency Department of our hospital for emergent evaluation of paraplegia and oliguria, from the local hospital of the nearby town, where he was admitted complaining from sudden, painless, progressive bilateral leg weakness and oliguria 4 days earlier. He gave no history of hypertension, diabetes mellitus or hyperlipidaemia, and had a negative family history of aortic diseases. On initial evaluation, the patient had a blood pressure of $131 / 71 \mathrm{~mm} \mathrm{Hg}$. His oral temperature was $36.4^{\circ} \mathrm{C}$, pulse rate was 82 beats/min and respiratory rate was $20 / \mathrm{min}$. He presented complete flaccid paraplegia with oliguria (urinary output $<400 \mathrm{ml} / \mathrm{d}$ ) and urinary retention, loss of pain and temperature sensation, vibration and position sense below the TH7 level bilaterally. Other general physical examinations were unremarkable. Laboratory tests showed a white blood cell count of $19.80 \times 10^{9} / 1$, haemoglobin concentration of $109 \mathrm{~g} / 1$, blood urea nitrogen concentration of $50 \mathrm{mmol} / \mathrm{l}$, blood creatinine concentration of $820 \mu \mathrm{mol} / \mathrm{l}$, sodium concentration of $114 \mathrm{mmol} / \mathrm{l}$, and potassium concentration of $4.6 \mathrm{mmol} / \mathrm{l}$. The liver function tests were normal and other observations were unremarkable. Later thoracic and lumbar MRI revealed swelling of thoracolumbar spinal cord, with no enhancement on T1-weighted images (wi) and increased signal on T2-wi at the TH9-TH12 levels, suggesting cord ischaemia. At the same MR sequences, the double lumen of the descending aorta involving bilateral renal arteries indicated dissection in both sagittal and axial images. The diagnosis of Stanford type B acute aortic dissection was confirmed. When patients present with or develop signs and symptoms of paraplegia without obvious cause, aortic dissection should be considered, even without the presence of characteristic thoracic pain.

Conclusions A 67-year-old man was transferred to the Emergency Department of our hospital for emergent evaluation of paraplegia and oliguria, from the local hospital of the nearby town, where he was admitted complaining from sudden, painless, progressive bilateral leg weakness and oliguria 4 days earlier. He gave no history of hypertension, diabetes mellitus or hyperlipidaemia, and had a negative family history of aortic diseases. On initial evaluation, the patient had a blood pressure of $131 / 71 \mathrm{~mm} \mathrm{Hg}$. His oral temperature was $36.4^{\circ} \mathrm{C}$, pulse rate was 82 beats/min and respiratory rate was $20 / \mathrm{min}$. He presented complete flaccid paraplegia with oliguria (urinary output $<400 \mathrm{ml} / \mathrm{d}$ ) and urinary retention, loss of pain and temperature sensation, vibration and position sense below the TH7 level bilaterally. Other general physical examinations were unremarkable. Laboratory tests showed a white blood cell count of $19.80 \times 10^{9} / 1$, haemoglobin concentration of $109 \mathrm{~g} / \mathrm{l}$, blood urea nitrogen concentration of $50 \mathrm{mmol} / \mathrm{l}$, blood creatinine concentration of $820 \mu \mathrm{mol} / \mathrm{l}$, sodium concentration of $114 \mathrm{mmol} / \mathrm{l}$, and potassium concentration of $4.6 \mathrm{mmol} / \mathrm{l}$. The liver function tests were normal and other observations were unremarkable. Later thoracic and lumbar MRI revealed swelling of thoracolumbar spinal cord, with no enhancement on T1-weighted images (wi) and 


\section{ABSTRACTS}

increased signal on T2-wi at the TH9-TH12 levels, suggesting cord ischaemia. At the same MR sequences, the double lumen of the descending aorta involving bilateral renal arteries indicated dissection in both sagittal and axial images. The diagnosis of Stanford type B acute aortic dissection was confirmed. When patients present with or develop signs and symptoms of paraplegia without obvious cause, aortic dissection should be considered, even without the presence of characteristic thoracic pain. 